\title{
Racial and socioeconomic disparities in sexual activity among older married Malaysians
}

\begin{abstract}
Sexuality as an important part of life has not been well studied in Malaysia, particularly among older adults. The main aim of this study was to investigate the racial and socioeconomic differences in sexual activity among older married Malaysians. Data for this study consisting of 1036 older married adults aged 60 years and older were obtained from the nationwide community-based cross-sectional survey entitled ñDeterminants of Wellness among Older Malaysian: A Health Promotion Perspectiveò, conducted in 2010. Data were analyzed using SPSS version 21 for Windows. The results showed that 57.3\% (95\% CI: $54.3 \mathrm{i} 60.3)$ of the respondents (61.6\% of men and $50.6 \%$ of women) had engaged in sexual intercourse during the last 12 months. The adjusted multivariate logistic regression analysis revealed that ethnicity and educational attainment were independently and significantly associated with sexual activity, after controlling for the possible confounding effects of chronic medical conditions and demographic characteristics. The findings from this study do support the notion that sexuality is a lifelong need and cultural teachings and formal education may have important role in maintaining the quality of sexuality in later life.
\end{abstract}

Keyword: Aged; Ethnicity; Sexual activity; Socioeconomic status 